\title{
REFORMAS NOS SISTEMAS DE SAÚDE ALEMÃO, FRANCÊS E BRITÂNICO
}

\author{
Reforms in German, French and British health systems \\ Reformas de los sistemas de salud alemán, francés y británico
}

\author{
Mariana Ribeiro Jansen Ferreira (OrcID) \\ Pontifícia Universidade Católica de São Paulo - PUC/SP - São Paulo (SP) - Brasil \\ Áquilas Nogueira Mendes (OrcID) \\ Faculdade de Saúde Pública - FSP/USP - São Paulo (SP) - Brasil \\ Pontifícia Universidade Católica de São Paulo - PUC/SP - São Paulo (SP) - Brasil
}

\section{RESUMO}

Objetivo: Mapear as reformas dos sistemas de saúde da Alemanha, França e Reino Unido, ao longo do período 1980 , 1990 e 2000, bem como apresentar as modificações recentes por meio da crise econômica de 2008/2009. Métodos: Trata-se de artigo original e ancora-se na abordagem do método da economia política através da descrição histórica dos processos de mudanças na mercantilização dos sistemas de saúde, com caráter qualitativo. Para a realização do exame da série de alterações dos sistemas de saúde, utiliza-se uma análise comparativa nos aspectos: mecanismos de contenção de custos; instrumentos de mercantilização; descentralização na gestão e alocação de recursos; regulamentação; financiamento; e cobertura. Resultados: Ao longo das décadas analisadas, os três sistemas de saúde experimentaram reformas que envolveram: novas formas de financiamento; ampliação de co-pagamentos, de seguros privados, de parcerias público-privadas, de terceirização de serviços auxiliares, atendimentos de pacientes privados na rede pública; incorporação do setor privado na oferta de serviços; gestão privada de instituições públicas; aumento da concorrência entre prestadores de serviços públicos e pagamento por resultados nos hospitais. Conclusão: As reformas geraram sistemas mais mercantilizados preocupados com os custos, mas em que a demanda social exigiu ampliação de cobertura com crescente uso de recursos fiscais para seu financiamento.

Descritores: Sistemas de Saúde; Economia e Organizações de Saúde; Reforma dos Serviços de Saúde.

\section{ABSTRACT}

Objectives: To map the reforms experienced in German, French and British health systems over the period 1980, 1990 and 2000, as well as to present the recent modifications by means of the economic crisis of 2008/2009. Methods: This is an original article based on the approach of the political economy method, through the historical description of change processes in the commodification of the health systems, with qualitative characteristics. For examination of the series of changes seen in the health systems, a comparative analysis is used, addressing these aspects: cost-containment mechanisms; instruments of commodification; decentralization of management and allocation of resources; regulation; financing; and coverage. Results: Over the analyzed decades, the three health systems experienced reforms that involved: new forms of financing; expansion of co-payments, private insurance, public-private partnerships, outsourcing of auxiliary services, private patient care in the public network; incorporation of the private sector in the provision of services; private management of public institutions; increased competition between public service providers; payment by results in hospitals. Conclusion: The reforms generated more commoditized and cost-oriented systems, but in which the social demand forced the extension of coverage, with increasing use of fiscal resources for their financing.

Descriptors: Health Systems; Health Care Economics and Organizations; Health Care Reform.

\section{RESUMEN}

Objetivo: Mapear las reformas de los sistemas de salud de Alemania, Francia y Reino Unido a lo largo de los años 80,90 y 2000 así como presentar los cambios recientes a través de la crisis económica de 2008/2009. Métodos: Se trata de un artículo original basado en el método de la economía política a través de la descripción histórica de los procesos de cambios de la mercantilización

Este artigo foi selecionado, corrigido e aprovado pela Comissão Científica do Fórum Internacional de Sistemas Universais de Saúde, seguindo suas normas e formatação. 
de los sistemas de salud con carácter cualitativo. Para la realización del análisis de los cambios de los sistemas de salud se utilizó un análisis comparativo de los siguientes aspectos: los mecanismos de contención de costes; los instrumentos de mercantilización; la descentralización de la gestión y el destino de los recursos; la reglamentación; la financiación y la cobertura. Resultados: A lo largo de las décadas analizadas, los tres sistemas de salud vivenciaron reformas que involucraron nuevas formas de financiación, la ampliación de co-pagos, de seguros privados, de colaboración público-privados, de tercerización de los servicios auxiliares, la atención de pacientes privados en la red pública; la incorporación del sector privado para la oferta de servicios; la gestión privada de instituciones públicas; el aumento de la competencia entre los prestadores de servicios públicos y el pago a cambio de resultados en los hospitales. Conclusión: Las reformas llevaron a sistemas más mercantilizados preocupados con los costes pero la demanda social exigió la ampliación de la cobertura con el uso crecente de recursos fiscales para su financiación.

Descriptores: Sistemas de Salud; Economía en Atención de Salud y Organizaciones; Reforma de la Atención de Salud.

\section{INTRODUÇÃO}

Após a Segunda Guerra Mundial, diversos países ocidentais de renda elevada criaram uma ampla rede de proteção social. Este movimento está relacionado a uma série de fatores: forte pressão por parte dos trabalhadores; necessidade de legitimar o capitalismo, frente à URSS no contexto da Guerra Fria; no caso europeu, o processo de reconstrução pós-guerra; especificidades de produção fordista, baseada na produção e no consumo em massa(1).

A partir desse específico momento histórico, constituíram-se os Estados de Bem-Estar Social, sendo os sistemas de saúde parte importante dessa rede de proteção. Os sistemas criados foram capazes de gerar, entre as décadas de 1950 e 1970, acesso amplo e universal aos serviços de saúde. Juntamente a melhorias nas condições sanitárias dos grandes centros urbanos, proporcionaram forte ampliação na expectativa de vida e nas condições gerais de saúde ${ }^{(2-4)}$.

Cabe ressaltar que os sistemas de saúde possuíam diferenças importantes, refletindo processos históricos particulares prévios. Ao se considerar três países para estudo, como Alemanha, França e Reino Unido, conseguese destacar algumas diferenças centrais no desenho de seus sistemas de saúde do pós-guerra ${ }^{(1-4)}$. Em relação à Alemanha, destaca-se um sistema de saúde corporativo, centrado em Caixas de Saúde geridas e financiadas por trabalhadores e empregadores. Sistema voltado para o mercado de trabalho, incorporando categorias "não-tradicionais" ao longo do tempo. No caso da França tem-se um sistema de saúde corporativo, mas com ampla regulação e gestão estatal, definindo contribuições sociais e alocação de recursos, com Caixa centralizada. No tocante ao Reino Unido, salienta-se um sistema de saúde público direcionado a toda população, com gestão e fornecimento de serviços direto pelo Estado (com nacionalização do sistema hospitalar) e financiamento mediante impostos ${ }^{(2,4)}$.

A partir de meados da década de 1970, no entanto, a retração no ritmo de crescimento econômico, com aumento na taxa de desemprego e na taxa de inflação, passou a pressionar as condições econômicas e sociais. Ao longo dos anos 1980, 1990 e 2000, os sistemas de saúde universais passaram por uma série de alterações que tinham como meta reduzir seus custos e ampliar sua "eficiência", incluindo seis tendências como a inserção de mecanismos de contenção de custos e o crescimento do setor privado e da adoção de princípios de mercado nos sistemas de saúde: a mercantilização. Além disso, as reformas incluíram também alterações em direções distintas: descentralização (parcial) na gestão e alocação de recursos nos sistemas; aumento da regulamentação; ampliação da base de financiamento; ampliação na cobertura de acesso ${ }^{(4,5)}$.

Sendo assim, este trabalho tem como objetivo mapear as reformas dos sistemas de saúde da Alemanha, França e Reino Unido, ao longo do período 1980, 1990 e 2000, bem como apresentar alterações recentes por meio da crise econômica de 2008/2009. Ainda que seja prematuro apontar as consequências das reformas em termos de acesso e equidade, é importante explicitar o escopo das mudanças e seus potenciais riscos à efetiva universalidade que marcou historicamente esses sistemas de saúde ${ }^{(4)}$.

\section{MÉTODOS}

O trabalho é original e ancora-se na abordagem do método da economia política através da descrição histórica dos processos de mudanças na mercantilização dos sistemas de saúde da Alemanha, França e Reino Unido, com caráter qualitativo ${ }^{(6,7)}$. Os dados deste trabalho tiveram como base as informações disponíveis na literatura internacional sobre as reformas dos sistemas de saúde nos três países, coletadas em pesquisa bibliográfica realizada na França durante o primeiro semestre do ano de 2016, no âmbito de estágio no exterior dos autores. 
Realizou-se uma análise comparativa sobre como a adoção de mecanismos de mercado provocou efeitos nos três sistemas de saúde distintos ${ }^{(1)}$. Alemanha, França e Reino Unido foram selecionados por apresentarem características semelhantes que permitem a comparação em três temáticas: como se deu o desenvolvimento de seus sistemas de saúde, após a Segunda Guerra Mundial; as mudanças da crise das décadas 1970/1980; e os desafios no contexto do mercado comum europeu. Por sua vez, são consideradas as diferenças importantes que contribuem para compreender como desenhos institucionais distintos respondem à introdução de mecanismos de mercado.

Para o trabalho comparativo das políticas públicas desenvolvidas pelos três países foram considerados alguns aspectos essenciais, de acordo com a literatura especializada nessa temática: a análise de como as políticas públicas mudam; a utilização do trabalho comparativo em si e a avaliação de sua relevância; a realização da análise considerando um período relativamente longo no tempo (30 anos ou mais) ${ }^{(1)}$.

\section{RESULTADOS E DISCUSSÃO}

Embora as reformas dos sistemas de saúde da Alemanha, França e Reino Unido não sejam lineares, há características comuns que podem ser destacadas. Nesse sentido, é possível realizar uma periodização das reformas, mostrando como elas mudaram ao longo das décadas e aprofundaram as alterações nos sistemas ${ }^{(2,4)}$.

Na década de 1980, frente à crise econômica e à crescente pressão para redução dos gastos (sociais), os países realizaram adaptações em suas economias e em seus Estados de bem-estar social. O mesmo ocorreu com seus sistemas de saúde. Sendo assim, a década é marcada por três tipos de medidas: políticas de contenção de custos; ampliações nas taxas de contribuições sociais; implementação de novos mecanismos de co-pagamento. As alterações ocorreram sem modificar características estruturais dos sistemas. Mesmo a criação de novos copagamentos não gerou mudanças institucionais, considerando que o co-pagamento já estava presente nos três sistemas e seu impacto continuou sendo mitigado pela existência de isenções ${ }^{(4,5)}$.

No entanto, nas décadas de 1990 e 2000, as alterações são mais profundas. Os governos modificaram a composição do financiamento dos sistemas de saúde e transformaram a oferta dos serviços de saúde, com crescente incorporação de mecanismos de mercado nas instituições públicas, aumento da participação privada e ampliação da esfera regulatória ${ }^{(4,5)}$.

A década de 1990 foi marcada, assim, por mudanças na estrutura de financiamento; uma primeira fase da mercantilização (com adoção de mecanismos de mercado e ampliação da participação privada nos sistemas); e descentralização (marcadamente na França e no Reino Unido, sistemas de saúde caracterizados na sua constituição pela forte centralização) $)^{(2,3)}$. Ainda que o setor privado sempre tenha feito parte dos sistemas de saúde nos três países, a partir dessa década, sua participação se torna maior, em termos de financiamento e provisão de serviços, incorporando no setor público, principalmente hospitalar, a lógica de gestão privada (new public management) ${ }^{(3)}$.

Nos anos 2000, identificam-se reformas com um duplo movimento: de um lado, aprofundamento das mudanças estruturais, com ampliação da mercantilização e da maior regulamentação dos sistemas. Por outro lado, há correções de aspectos negativos de parte das alterações da década de 1990 e mudança no enfrentamento da situação de baixo crescimento econômico e elevado desemprego ${ }^{(4)}$.

A fim de garantir acesso aos sistemas nestas circunstâncias, a França ampliou a universalização do acesso e o Reino Unido expandiu os recursos, reduzindo as filas de espera( ${ }^{(4,5)}$. Pode-se afirmar que a própria crise econômica e as reformas realizadas como reação pressionaram pela garantia de acesso aos sistemas de saúde fora dos moldes do pós-guerra(2,4).

Sendo assim, a década de 2000 é marcada por: ampliação da participação privada e da incorporação de mecanismos de mercado (identificada nesse trabalho como segunda fase de mercantilização); aumento na regulamentação (constituída em contrapartidada, justamente, da maior participação privada nos sistemas); ampliação da transformação do paciente em "consumidor" de serviços de saúde; ampliação do acesso aos sistemas ${ }^{(4)}$.

A partir da periodização apresentada, identificam-se seis tendências que caracterizam as reformas no período de 1980 a 2010. A primeira é a introdução de mecanismos de controle de custos ${ }^{(5)}$.

Os três países implementaram diferentes mecanismos de controle com medicamentos: estabelecimento de uma "lista positiva" definindo quais medicamentos seriam pagos (Reino Unido), criação de uma "guia de referências" de medicamentos a serem prescritos (França), imposição de tetos aos preços (Alemanha), elevação nos co-pagamentos sobre medicamentos (nos três países) ${ }^{(2,8,9)}$.

A França e a Alemanha, países cuja remuneração aos prestadores de serviços era anteriormente pautada pela quantidade de pacientes atendidos pelos médicos e pelo uso dos leitos per diem, implementaram, entre o final da 
década de 1970 e início dos anos 1990, tetos orçamentários ("envelope global de despesas") em seus setores hospitalares. No caso francês, o orçamento pré-definido foi adotado nos hospitais públicos e filantrópicos, mas não nos hospitais privados que prestavam serviços à Seguridade Social. Estes continuaram a ser remunerados com base na taxa de utilização per diem ${ }^{(2,4,10)}$.

A França implementou uma mudança mais profunda na década de 1990, com a aprovação da Lei de Financiamento da Seguridade Social (LFSS), em 1996, como parte do Plano Juppé. Dentro do Orçamento da Seguridade, a alocação dos recursos à saúde passou a ser definido a partir do Objetivo Nacional de Despesas de Seguro Saúde, que definia o orçamento geral para o setor de saúde e sua distribuição em subsetores de cuidado(2,11).

Os mecanismos de controle de custos não foram capazes, no entanto, de reduzir de forma significativa a pressão para mais recursos nos sistemas de saúde, tendo em vista o envelhecimento populacional e os custos envolvidos na incorporação de novas tecnologias no setor. Isso se torna claro na Tabela I, que explicita a alocação crescente de recursos públicos na saúde.

Tabela I - Gasto em saúde, indicadores diversos, Alemanha, França e Reino Unido, 1970 a 2015.

\begin{tabular}{|c|c|c|c|c|c|c|c|c|c|c|}
\hline & 1970 & 1975 & 1980 & 1985 & 1990 & 1995 & 2000 & 2005 & 2010 & 2015 \\
\hline \multicolumn{11}{|c|}{ Gasto total em saúde, em \% PIB } \\
\hline Alemanha & 5,7 & 8,0 & 8,1 & 8,5 & 8,0 & 9,5 & 9,8 & 10,3 & 11,0 & 11,2 \\
\hline França & 5,2 & 6,1 & 6,7 & 7,6 & 8,0 & 9,8 & 9,5 & 10,2 & 10,7 & 11,1 \\
\hline Reino Unido & 4,0 & 4,9 & 5,1 & 5,1 & 5,1 & 5,7 & 6,0 & 7,2 & 8,5 & 9,9 \\
\hline \multicolumn{11}{|c|}{ Gasto total em saúde per capita, em paridade do poder de compra, preços constantes (US\$) } \\
\hline Alemanha & 263 & 567 & 960 & 1.400 & 1.757 & 2.251 & 2.709 & 3.331 & 4.413 & 5.353 \\
\hline França & 193 & 365 & 655 & 1.012 & 1.412 & 2.053 & 2.505 & 3.124 & 3.872 & 4.530 \\
\hline Reino Unido & 142 & 265 & 429 & 629 & 852 & 1.143 & 1.565 & 2.337 & 3.041 & 4.125 \\
\hline \multicolumn{11}{|c|}{ Gasto público em saúde, em \% gasto total em saúde } \\
\hline Alemanha & 71,4 & 78,1 & 77,8 & 76,6 & 75,4 & 81,7 & 79,4 & 76,3 & 83,5 & 84,5 \\
\hline França & 74,9 & 77,5 & 79,6 & 78,0 & 76,0 & 79,1 & 78,9 & 78,7 & 78,4 & 78,9 \\
\hline Reino Unido & 86,4 & 90,9 & 89,5 & 86,1 & 84,3 & 84,1 & 79,3 & 81,3 & 83,1 & 79,7 \\
\hline
\end{tabular}

Fonte: $\operatorname{OCDE}^{(10)}$

Sendo assim, uma segunda alteração importante foi com relação à ampliação na base de financiamento. Em países com financiamento via contribuições sociais, houve aumento da alíquota de contribuição. Entre 1970 e 1997, a taxa de contribuição social total (paga por trabalhadores e empregadores) destinada à saúde, em relação aos salários, aumentou de $8,2 \%$ para $13,5 \%$, na Alemanha, e de $15,75 \%$ para $19,6 \%$, na França ${ }^{(12-14)}$. Além disso, outras fontes de recursos também passaram a financiar os sistemas de saúde nestes dois países: na Alemanha, ocorreu o direcionamento de uma parcela do imposto sobre cigarros à saúde, gerando, entre 2004 e 2008, uma média de $€ 2,34$ bilhões ao ano ${ }^{(15,16)}$.

Esse processo é mais amplo na França. Desde 1983, o governo passou a alocar parcela dos impostos arrecadados sobre o cigarro e o tabaco à saúde. Em 1990, o governo criou a Contribuição Social Generalizada (CSG). A CSG incidia, inicialmente, sobre três tipos de rendas: os rendimentos do trabalho e as rendas compensatórias (seguro desemprego, seguro invalidez, previdência); os rendimentos patrimoniais/de capital; e os rendimentos provenientes de aplicações financeiras. Posteriormente, passou a incidir também sobre a receita de loterias e sobre as vendas e gastos com propaganda da indústria farmacêutica. Em sua criação, a taxa da CSG era de 1,1\% sobre todos os rendimentos, aumentando para 2,4\% (1993), 3,4\% (1995) e, finalmente, 7,5\% (1996), com a implementação da Lei de Financiamento da Seguridade Social (LFSS). Dos 7,5\%, 5,25\% são destinados à saúde e representava, em 2012, 36\% de todos os recursos do setor ${ }^{(10,17)}$.

No entanto, a CSG não aumentou o volume de recursos destinados ao financiamento do sistema de saúde, dado que foi acompanhada de uma redução nas contribuições sociais. A contribuição social paga pelos empregadores permaneceu a mesma $(12,8 \%$ ), mas a parcela dos trabalhadores diminuiu de $6,8 \%$ para $0,75 \%$ (recurso destinado especificamente para financiar o auxílio-doença, durante afastamento do trabalho). A maior mudança gerada pela criação da CSG foi a inclusão dos rendimentos patrimoniais/de capital e provenientes de aplicações financeiras no financiamento da Seguridade Social, principalmente da saúde ${ }^{(10,17)}$. 
No caso do Reino Unido, os recursos orçamentários foram ampliados ao longo da década de 2000. Entre 2000 e 2010 , os recursos para o NHS aumentaram mais de $6 \%$, levando o gasto público com saúde a crescer de $5 \%$ para $7,2 \%$ do PIB, maior ritmo de crescimento, no período, entre todos os países da $\operatorname{OCDE}^{(8,12)}$.

Além de alterações financeiras, os sistemas de saúde passaram por importantes modificações em suas gestões. Destacamos como terceira mudança a descentralização (parcial) da gestão e alocação dos recursos, caracterizada por um movimento dual. De um lado, a transferência, ao menos parcial, das responsabilidades da esfera nacional para a esfera regional/local, buscando maior eficiência na alocação dos recursos. Por outro lado, no controle de recursos, houve um movimento inverso, com maior centralização das decisões ${ }^{(18)}$.

No Reino Unido, o duplo movimento ocorreu já na década de 1980, quando as autoridades locais de saúde foram substituídas por distritos de saúde, em uma divisão regional muito maior. Nos anos 1990, somou-se a esse processo o aumento de poder decisório a outros agentes sociais, principalmente após a instituição do "mercado interno", em 1991. No final dessa década, o governo Blair realizou o maior processo de descentralização, com a devolução das responsabilidades por seus sistemas de saúde a cada país que compõe o Reino Unido, ampliando a autonomia da Escócia, Irlanda do Norte e País de Gales frente às decisões inglesas ${ }^{(8,18)}$.

Nos anos 2000, o poder regional voltou a ser reforçado na Inglaterra, com os cuidados ambulatoriais (na forma dos Primary Care Trusts - PCTs) e hospitalares (foundation trusts) mais claramente subordinados à esfera local e à lógica de mercado ${ }^{(8)}$. Na reforma de 2012, por sua vez, há nova centralização: os PCTs foram substituídos por Clinical Commissioning Groups (CCGs), que contratualizam todos os serviços de saúde necessários para as pessoas nas suas listas, subordinados ao Departamento de Saúde ${ }^{(17)}$.

No caso francês, o Plano Juppé, de 1996, criou as Agências Regionais de Hospitalização (ARH) $)^{(9)}$. As ARHs deveriam, a partir dos orçamentos e diretrizes definidas nacionalmente para sua região, alocar os recursos e responsabilidades entre os hospitais de sua região. Em 2003, essa regionalização foi ampliada, com as próprias ARHs definindo as diretrizes locais para alocação do dinheiro, mediante os "esquemas regionais de organização dos cuidados". Na reforma de 2010, a descentralização teve prosseguimento, com a substituição das ARH pelas Agências Regionais de Saúde (ARS). As ARS, além das funções das ARH, passaram a realizar também a gestão ambulatorial, anteriormente exercidas por três diferentes instituições públicas nacionais ${ }^{(19,20,21)}$.

Por outro lado, parte das responsabilidades que antes eram regionais foram transferidas para a esfera central. Primeiro, as políticas de garantia de acesso aos serviços de saúde à população mais pobre, que era departamental (regiões francesas), foi centralizada com a criação da Cobertura Universal para Doenças (CMU), em 1999. Outro exemplo refere-se às campanhas de prevenção e cuidados contra as doenças venéreas, lepra, tuberculose e câncer - de responsabilidade departamental até 2004, quando se tornam responsabilidade do governo central( ${ }^{(0,20)}$.

Na Alemanha, a descentralização sempre esteve presente em seu sistema de seguro social, vide o poder das Caixas de Saúde, que concentravam a gestão, organização e distribuição de recursos na esfera ambulatorial. A mudança importante, verificada nas décadas de 1990 e 2000, foi um maior controle do governo central sobre os dispêndios das Caixas, centralizando decisões como a alíquota da contribuição social. Ainda assim, o sistema manteve sua lógica descentralizada de organização(13,18).

Nos três países, a maior descentralização dos sistemas nas décadas de 1990 e 2000 foi relativa, dado que veio acompanhada de um maior controle da esfera central dos gastos totais do setor, assim como de um aumento na participação de instituições independentes na regulamentação dos serviços de saúde. Isso aponta para a quarta tendência que se identificou: o aumento da regulamentação( ${ }^{(18)}$.

O Estado passou a regulamentar as negociações entre Caixas e prestadores de serviços tanto na Alemanha (1993) como na França (1996), em caso de descumprimento de parâmetros legais - principalmente no que dizia respeito ao cumprimento dos tetos orçamentários. No caso francês, a lei de financiamento de Seguridade Social tornou essa interferência mais direta, dado que passou a ser função do Parlamento a definição de recursos no setor. $\mathrm{Na}$ Alemanha, essa interferência se ampliou, principalmente nos anos 2000, com a retirada da liberdade das Caixas em definirem sua taxa de contribuição social (e a criação de uma taxa única definida pelo Estado) e com a criação de um Fundo único de Financiamento, em 2007, com a introdução de novos critérios para sua redistribuição que, além de idade, incluiu tipos de morbidade(18,21,22).

Nos três países, a criação destas agências representou a internalização da lógica de eficiência da gestão, ao incorporar a adoção de critérios de eficiência para definição de procedimentos e medicamentos a ingressarem nos sistemas $^{(13)}$.

Na França, o governo criou, em 1996, a Agência Nacional da Acreditação e Avaliação em Saúde, que utiliza tanto critérios clínicos (como já existia) quanto explicitamente econômicos ${ }^{(21,23)}$. No caso alemão, a tendência de 
criação de agências regulatórias só foi efetivamente implementada na reforma de 2003, com a criação do Instituto de Qualidade e Eficiência Econômica em Saúde, uma instituição independente que tem como prerrogativa difundir normas terapêuticas e instrumentos para avaliação, como consultor técnico para as decisões das Caixas ${ }^{(13,22)}$.

Em termos de agências regulatórias, no entanto, as maiores mudanças ocorreram no Reino Unido, mais especialmente na Inglaterra. Os órgãos criados foram de quatro tipos: instituições responsáveis pela regulamentação da estrutura local de saúde; órgãos para análise da relação custo-efetividade para inclusão de novos medicamentos e tecnologia no NHS; instituições para análise de qualidade de todos os provedores e monitoramento a partir de padrões nacionais de qualidade e segurança; regulamentação específica dos provedores de serviços privados ou autônomos atuando no NHS. Quanto a este último, o Monitor foi desenvolvido como uma instituição regulatória independente, tendo se tornado, rapidamente, o órgão mais importante de regulamentação hospitalar (após 2003, com o estabelecimento dos hospitais como instituições públicas de direito privado) e das instituições privadas independentes contratadas para prestar serviços junto ao NHS. Em 2009, a instituição ampliou ainda mais sua esfera de atuação, passando a operar como "regulador econômico", tendo como prerrogativa que tanto a cooperação como a concorrência são desejáveis no sistema de saúde ${ }^{(19,21,24)}$.

Uma quinta tendência importante foi a ampliação na cobertura, garantindo maior acesso aos sistemas de saúde. Alemanha e França criaram sistemas de saúde no pós-guerra em torno do trabalhador assalariado. Entre as décadas de 1950 e 1970, ampliaram a inclusão para trabalhadores rurais, profissionais liberais e estudantes, além de expandirem aos desempregados. Com a crise econômica dos anos 1970 e o aumento do desemprego e do trabalho precário, o risco de exclusão dos sistemas tornou-se real. Na Alemanha, ainda que o seguro social, somado aos mecanismos assistenciais, garantisse quase plena cobertura, estimava-se, em 2006, entre 80 a 300 mil o número de alemães sem qualquer tipo de cobertura à saúde. Sendo assim, em 2007, tornou-se obrigatória a adesão a alguma forma de seguro (social ou privado) para toda a população(2,16).

Na França, o governo criou, em 2000, a Cobertura Universal para Doenças (CMU), seguida da implementação da Cobertura Universal para Doenças - complementar (CMU-C), em 2003. A CMU e a CMU-C permitiram o acesso ao sistema de saúde a toda população abaixo de determinado nível de renda, que não pagava a contribuição social - em outubro de 2014, o teto para acesso a CMU e CMU-C gratuita era de cerca de $€ 800$ mensais por pessoa (o valor varia conforme o tamanho da família). Por sua vez, a CMU-C permitiu acesso sem ter que arcar com o alto copagamento ou com o pagamento do seguro complementar. Em 2005, essa política foi complementada pela criação da Ajuda Complementar à Saúde (ACS), que visa atingir a parcela da população com renda mais elevada do que o teto do CMU-C, mas que, ainda assim, não possui recursos suficientes para pagar um seguro complementar. A ACS foi definida a partir do teto da CMU-C, com um acréscimo de até $35 \%$ na renda. Ao invés do pagamento de $100 \%$ de um seguro complementar, como na CMU-C, a ACS fornece um "vale", cujo valor varia conforme a idade da pessoa ${ }^{(9,17,25)}$.

A sexta tendência identificada foi a crescente mercantilização, entendida como o aumento da participação direta do setor privado no financiamento e prestação de serviços de saúde, além da crescente incorporação de princípios privados (como a eficiência) nos sistemas públicos de saúde ${ }^{(13)}$.

Inicialmente, entre meados dos anos 1970 e os anos 1980, os três países expandiram seus mecanismos de co-pagamento, inclusive no caso de internação hospitalar e em outros cuidados considerados não essenciais (principalmente oftalmológicos e dentários). Nesse período, o Reino Unido iniciou a terceirização de serviços não clínicos $^{(9,13,26)}$.

A preocupação com a eficiência aprofundou-se na década de 1990. Os três países incentivaram a concorrência enquanto mecanismo de alocação eficiente dos recursos, com a premissa de que viabilizaria a preservação - e até ampliação - do acesso aos sistemas. Esse processo foi mais acentuado na Alemanha, com a implementação de concorrência entre as Caixas de Saúde, e no Reino Unido, mediante a adoção do "mercado interno". Ademais, o Reino Unido implementou parcerias público-privadas (PPPs) para o financiamento de estruturas hospitalares, com mecanismos semelhantes sendo adotados na Alemanha e França, nos anos $2000^{(27)}$.

Por sua vez, a Alemanha como o Reino Unido expandiram a participação de hospitais privados na oferta dos serviços: na Alemanha, por meio da privatização de instituições públicas municipais e, na Inglaterra, mediante a contratação de instituições privadas (setor independente) para prestar serviços junto ao $\mathrm{NHS}^{(27)}$.

As características de mercado também aparecem no aumento da concorrência entre os provedores de serviços no caso inglês, assim como na ampliação da liberdade tarifária por parte dos médicos franceses. A Seguridade Social francesa convenciona, por meio de contratos entre os médicos e as Caixas de Saúde, as tarifas que os profissionais recebem por atendimento. A pressão da medicina liberal fez com que os médicos sempre tenham podido cobrar 
dos pacientes valores maiores do que a tarifa definida pela Seguridade Social; porém, nestes casos, o paciente só é reembolsado até o teto do valor definido pela Seguridade, devendo arcar com o valor restante ou contratar um seguro privado complementar para arcar com esta diferença. Ao longo dos anos 1990 e 2000, o percentual de medicamentos cobrando tarifas mais elevadas tornou-se crescente, chegando a 95\% dos cirurgiões atuando com tarifa diferenciada ${ }^{(28,29)}$.

Destaca-se, ainda, nos três países, a ampliação na adoção da gestão de mercado (new public management), com o estabelecimento de metas de custo-eficiência na alocação de recursos e a implementação de sistemas profissionais, e do pagamento por procedimento, na modalidade DRG (Diagnosis Related Group), nos hospitais ${ }^{(4)}$.

O grau de profundidade da adoção de mecanismos de mercado e da incorporação do setor privado gerou mudanças importantes na organização e na oferta dos serviços nos três sistemas de saúde. Tendo em vista a diversidade de instrumentos de mercado adotados e a maior participação direta do setor privado, sumarizam-se as mudanças em categorias de mercantilização, conforme apresentado no quadro I.

Quadro I - Tipos de mercantilização nos sistemas de saúde alemão, francês e britânico. 1980 - 2015.

\begin{tabular}{|c|c|c|c|}
\hline \multicolumn{2}{|c|}{ Mercantilização explícita } & \multicolumn{2}{|l|}{ Mercantilização implícita } \\
\hline Financiamento & Oferta de serviços & Financiamento & Oferta de serviços \\
\hline $\begin{array}{l}\text { Pagamento direto / } \\
\text { co-Pagamento }\end{array}$ & Tercerização de serviços auxiliares & Parceria público-privada & Nova gestão pública \\
\hline Seguro Privado & $\begin{array}{l}\text { Atendimento de pacientes privados } \\
\text { na rede pública } \\
\text { Incorporação setor privado na oferta } \\
\text { de serviços }\end{array}$ & $\begin{array}{l}\text { Ampliação concorrência } \\
\text { - Caixas de saúde }\end{array}$ & $\begin{array}{l}\text { Aumento da concorrência entre } \\
\text { prestadores de serviço (públicos) }\end{array}$ \\
\hline & $\begin{array}{l}\text { Gestão privada de instituições públicas } \\
\text { Privatização (stricto sensu) } \\
\text { Fusões e aquisições }\end{array}$ & & Pagamento por resultado - médicos \\
\hline
\end{tabular}

As seis tendências apresentadas foram bastante afetadas pela crise econômica internacional, que se iniciou no mercado imobiliário norte-americano em 2007, configurando-se em uma profunda crise de dívida pública nos Estados europeus, a partir de 2008-2009(4,30).

Entre 2008 e 2016, a dívida pública alemã cresceu de $65 \%$ para $68 \%$ do PIB (tendo chegado ao pico de $77 \%$ em 2012), a francesa de $68 \%$ para $97 \%$ do PIB e no caso britânico de $52 \%$ para $88 \%$ do $\mathrm{PIB}^{(30)}$. A forte ampliação do endividamento dos países europeus, embora diretamente relacionada com as políticas de reestruturação do sistema financeiro afetado pela crise, gerou novas pressões sobre as políticas e os dispêndios sociais ${ }^{(31,32)}$.

A forte alocação de recursos públicos para o financiamento de políticas sociais, como a saúde, a previdência, o seguro-desemprego, entre outras, seria o responsável por elevadas alíquotas de impostos e contribuições sociais, impactando na estrutura de custos das empresas e na capacidade de financiamento dos Estados-nacionais ${ }^{(31,32)}$.

Em 2010, o Departamento de Saúde inglês definiu uma taxa de crescimento de recursos para o NHS, em termos reais, de apenas $0,1 \%$ a.a., entre 2011 e 2015. O governo pressionou para que houvesse uma efetiva redução: ao longo destes cincos anos, os gastos hospitalares foram reduzidos em $7 \%$, em termos reais. Na França, apenas para o ano de 2013, a meta do governo era uma redução de $€ 2,4$ bilhões nos gastos com saúde, $73 \%$ por meio de redução nos gastos ambulatoriais, principalmente com medicamentos ${ }^{(33,34)}$.

Ao mesmo tempo, países como a Alemanha e a França buscaram expandir fontes fiscais para o financiamento dos sistemas de saúde. O governo francês ampliou as alíquotas de impostos sobre produtos considerados prejudiciais à saúde, assim como o percentual arrecadado direcionado ao setor: cigarro (em 2011), refrigerante (2012) e cerveja (2013). Além disso, novas fiscalidades foram criadas sobre lucro e grandes salários. Em 2009, uma nova contribuição social (forfait social sur l'épargne salariale) foi criada, inicialmente com incidência de $2 \%$ sobre a participação nos lucros, até atingir $20 \%$ em 2012, com $25 \%$ do total da receita obtida destinada à saúde. Em 2013, foi criado um imposto adicional sobre os salários anuais acima de $€ 150.000$, com os recursos destinados à Seguridade Social ${ }^{(34)}$.

Na Alemanha, o governo aumentou as transferências fiscais ao Fundo único de Saúde, responsável por centralizar, desde 2009, os recursos das contribuições sociais. Entre 2009 e 2013, transferiu $€$ 63,5 bilhões para o Fundo. Por outro lado, no mesmo período, o governo retirou uma das características centrais do financiamento 
do sistema alemão, que era a paridade de contribuição entre trabalhadores e empregadores. Desde 2010, a taxa nacional de $15,5 \%$ sobre os salários é repartida em $7,3 \%$ para os empregadores e $8,2 \%$ para os trabalhadores (a diferença de $0,9 \%$ refere-se ao financiamento dos cuidados dentários, que passaram a ser integralmente financiados pelos trabalhadores). Além disso, eliminou o limite para a cobrança de um valor adicional sobre os trabalhadores, em casos em que as Caixas de Saúde às quais estejam filiados apresentem déficits ${ }^{(33,35)}$.

Outro importante aspecto no que diz respeito ao financiamento, os co-pagamentos aos usuários do sistema, vem sendo majorados na França. O governo ampliou, em 2010-2011, a quantidade de medicamentos sem cobertura pela Seguridade Social, além de diminuir o percentual reembolsado pelo sistema para medicamentos considerados de baixa e média efetividade. Além disso, elevou o co-pagamento por dia de internação hospitalar. Por outro lado, na Alemanha e no Reino Unido não ocorreram aumentos de co-pagamento nesse período. Na Alemanha, o co-pagamento de $€ 10$ por consulta médica (não recomendada pelo clínico geral), criada em 2004, foi abolido em 2012; no caso do Reino Unido, enquanto na Inglaterra não se alteraram os co-pagamentos de medicamentos e cuidados dentários e oftalmológicos existentes, na Escócia e no País de Gales se extinguiu qualquer forma de co-pagamento(19,34,35).

As mudanças realizadas desde 2008 também envolveram um aprofundamento da busca por maior eficiência dos sistemas de saúde. A França ampliou a remuneração de hospitais e médicos ambulatoriais por meio do pagamento por resultado e tornou, em 2013, obrigatória a garantia do seguro privado por empresas com mais de 100 funcionários. Por sua vez, a Inglaterra aprofundou a competição interna ao NHS, principalmente após 2012, com a inclusão de hospitais privados como parte dos prestadores de serviço do sistema e ampliação da possibilidade de atendimentos de pacientes com planos privados de saúde na rede pública ${ }^{(10,24,36)}$.

O acesso aos sistemas de saúde, no entanto, explicitou o aspecto "multifacetado" da crise ${ }^{(34)}$. Em 2012, a Inglaterra modificou a universalidade automática de acesso ao NHS e passou a condicioná-la a que as pessoas constem das listas das instituições responsáveis pela gestão do atendimento ambulatorial. Apenas em casos de emergência hospitalar é que se poderia acessar o sistema de saúde sem registro prévio, o que pode prejudicar o acesso a cuidados, principalmente aos imigrantes ilegais ${ }^{(33,34,37)}$.

Por fim, a crescente imposição de mecanismos de controle de custos e a mercantilização foram impulsionadas pela perspectiva de que os altos custos da saúde eram responsáveis, em grande medida, pelo aumento dos déficits e da dívida pública. Frente a essa premissa, ambas as tendências estão presentes e se aprofundaram nas reformas implementadas nas últimas décadas: aumentos nos mecanismos de co-pagamento, redução de medicamentos cobertos pela rede pública, inclusão de critérios custo-eficiência e de gestão profissional em hospitais, ampliação de agentes privados dentro do sistema público, principalmente prestadores de serviço(33,35).

Ao mesmo tempo, os recursos públicos alocados mantiveram-se elevados, sempre correspondendo a mais de $75 \%$ dos recursos destinados à saúde nos três países. Mesmo frente a pressões econômicas, os países ampliaram, ao invés de reduzir, a cobertura de acesso à saúde, seja ampliando o escopo inicial de cobertura (como na Alemanha e na França, cujos sistemas foram perdendo suas características de serem atrelados ao trabalhador e ganhando universalidade independente do mundo do trabalho), seja aumentando substancialmente os recursos alocados no sistema (como no Reino Unido, em que as despesas públicas aumentaram de 5,1\% para 9,9\% do PIB, entre 1980 e 2015) $(33,34,37)$.

\section{CONSIDERAÇÕES FINAIS}

As reformas dos sistemas de saúde alemão, francês e britânico podem ser compreendidas enquanto parte de um processo de adaptação dos sistemas de saúde às transformações da realidade na qual foram forjados. As pressões contraditórias, em que de um lado tem-se uma concepção neoliberal (e, no caso europeu, coroada pelas exigências da União Europeia) que preconiza a redução de gastos sociais e, de outro lado, as demandas crescentes por recursos na saúde, frente a uma população envelhecendo, afetada pelo desemprego e com novas tecnologias consumindo recursos crescentes.

Ao longo das quatro décadas, as seis tendências apresentadas geraram sistemas mais mercantilizados e pautados na preocupação com os custos, mas em que a demanda social gerou ampliação de cobertura com crescente uso de recursos fiscais para financiar o sistema, com maior regulamentação e centralização na gestão dos recursos e descentralização na implementação das políticas (ainda que este movimento não seja homogêneo nos três países).

Além disso, a pressão "neoliberal" adquiriu nova força com a crise econômica de 2008-2009, que legitimou novas tentativas de redução dos dispêndios com os sistemas de saúde e controles mais restritos de acesso aos sistemas, reforçando características mercantis e trazendo dúvidas sobre a capacidade de os sistemas de saúde alemão, francês e britânico manterem-se, de fato, universais. 


\section{CONFLITOS DE INTERESSE}

Não houve conflitos de interesse durante o desenvolvimento da presente pesquisa.

Manuscrito baseado em Tese de doutoramento: Tendências e contratendências de mercantilização - as reformas dos sistemas de saúde alemão, francês e britânico. Faculdade de Saúde Pública da Universidade de São Paulo, 2016. $297 \mathrm{p}$.

\section{REFERÊNCIAS}

1. Palier B, Surel Y, Bossy L, Boussaguet L, Chabalier D, Devineau J; et al. L'explication du changement dans l'analyse des politiques publiques: identification, causes et mecanismes. Paris: L'Harmattan, coll. Logiques politiques; 2010.

2. Hassenteufel P, Palier $B$. Towards neo-bismarckian health care states? Comparing health insurance reforms in bismarckian welfare systems. Soc Policy Adm. 2007;41(6):574-96.

3. Hassenteufel P, Smyrl M, Genieys W, Moreno-Fuentes FJ. Programmatic Actors and the transformation of European Health care States. J Health Polit Policy Law. 2010;35(4):517-38.

4. Maarse $\mathrm{H}$. The privatization of health care in Europe: an eight-country analysis. J Health Polit Policy Law. 2006;31(5):981-1014.

5. André $C$, Batifoulier $P$, Jansen-Ferreira MR. Une nouvelle grille d'analyse des processus de privatisation en Europe. In: Abecassis P, Coutinet N, editores. Economie sociale: crises et renouveaux. Louvain la Neuve: Presses Universitaires de Louvain; 2015. p. 95-110.

6. Paulo J Neto, Braz M. Economia Política: uma introdução crítica. São Paulo: Cortez; 2006.

7. Minayo MCS. O desafio do conhecimento: pesquisa qualitativa em saúde. 13a ed. São Paulo: Hucitec; 2013.

8. Klein R. The new politics of the NHS. 4a ed. Prentice Hall: Pearson Education; 2001.

9. Tabuteau D. Démocratie Sanitaire: les nouveaux défis de la politique de santé. Paris: Odile Jacob; 2013.

10. Batifoulier P. Capital santé: quand le patient devient client. Paris: La Découverte; 2014.

11. Barbier JC. The French social protection system in the throes of reform (1975-2007). Documents de Travail du Centre d'Economie de la Sorbonne. Paris: Maison des Sciences Économiques; 2007.

12. OECD. OECD Health Statistics [Internet]. 2017; [acesso 2015 dez 17]. Disponível em: http://www.oecd.org/els/ health-systems/health-data.htm.

13. Busse R, Blümel M. Germany: Health system review. Health Syst Transit. 2014;16(2):1-296.

14. Théret $B$. The Place of national systems of social protection and political representation in socio-economic regulation. Evol Inst Econ Rev. 2011;7(2):177-208.

15. Gerlinger T, Schmucker R. A Long farewell to the Bismarck System: incremental change in the German Health Insurance System. German Policy Stud. 2009;5(1):3-20.

16. Hinrichs K. A Social insurance state withers away. Welfare state reforms in Germany - or: attempts to turn around in a cul-de-sac. In: Palier B, editor. A Long Goodbye to Bismarck? The Politics of Welfare Reform in Continental Europe. Amsterdam: Amsterdam University Press; 2010. p. 45-72.

17. Pellet R. L'évolution du financement de l'assurance-maladie: bilan et perspectives. Rev Econ Financ. 2004;76(3):87127.

18. Saltman RB, Bankauskaite V, Vrangbbaek K. editores. Decentralization in health care: strategies and outcomes. European Observatory on Health Care Systems Series. New York: McGraw-Hill; 2007.

19. Mays N, Dixon A, Jones L, editores. Understanding New Labour's market reforms of the English NHS. Londres: The King's Fund; 2011.

20. Catrice-Lorey A, Steffen M. Implementation of health care reforms in the bismarckian systems: unequal capacities. Revue française des affaires sociales. 2006;6-7:163-82. 
21. Hassenteufel P. Les systèmes de santé entre conceptualisation économique et reconceptualisation politique. Socio-logos [Internet]. 2014 [acesso em 2014 Mai 01]; 9. Disponível em: http://socio-logos.revues.org/2812

22. Stolpe M. Reforming Health Care - The German Experience. IMF Conference Public Health Care Reforms: Challenges and Lessons for Advanced and Emerging Europe [Internet]. Paris: Kiel Institute for the World Economy; 2011. [acesso em 2014 Jun 10]. Disponível em: https://www.imf.org/external/np/seminars/eng/2011/paris/pdf/ stolpe.pdf

23. Rochaix L, Wilsford $D$. State autonomy, policy paralysis: paradoxes of institutions and culture in the French health care system. J Health Polit Policy Law. 2005;30(1-2): 97-119.

24. Allen P. An economic analysis of the limits of market based reforms in the English NHS. BMC Health Serv Res. 2013;13(Suppl 1):S1.

25. Chadelat JF. Les enjeux du financement de la santé. Revue française d'administration publique. 2005;1(113):6573.

26. Webster C. The National Health Service: a political history. New York: Oxford University Press; 1998.

27. André C, Hermann C. Privatisation and Marketisation of Health Care Systems in Europe. In: Hermann C, Huffschmid J, editores. Privatization against the European Social Model: a critique of European policies and proposal for alternatives. Hampshire: Palgrave MacMillan; 2009. p. 129-144.

28. Poullier JP, Sandier S. France. Duke University Press. J Health Polit Policy Law. 2000;25(5):899-905.

29. Duval J. Le mythe du "trou de la Sécu". Paris: Raisons d'Agir; 2007.

30. Eurostat. General government gross debt. 2017; [acesso em 2018 Jan 9]. Disponível em: http://ec.europa.eu/ eurostat/web/products-datasets/-/teina230

31. Aglietta M. Zona do Euro. Qual o Futuro? São Paulo: Ideias \& Letras; 2013.

32. Aglietta M. Europe. Sortir de la crise et inventer l'avenir. Paris: Michalon Éditeur; 2014.

33. Giovanella L, Stegmüller K. Crise financeira europeia e sistemas de saúde: universalidade ameaçada? Tendências das reformas de saúde na Alemanha, Reino Unido e Espanha. Rio de Janeiro: Cad Saúde Pública. 2014;30(11): 1-19.

34. Brunn M, Brigham KB, Chevreul K, Hernández-Quevedo $C$. The impact of the crisis on the health system and health in France. In: Maresso A, Mladovsky P, Thomson S, Sagan A, Karanikolos M, Richardson E; et al., editores. Economic crisis, health systems and health in Europe. European Observatory on Health Systems and Policies, Observatory Studies Series. 2015;41:75-101.

35. Henke KD, Quentin W. Germany. In: Maresso A, Mladovsky P, Thomson S, Sagan A, Karanikolos M, Richardson E; et al., editores. Economic crisis, health systems and health in Europe. European Observatory on Health Systems and Policies, Observatory Studies Series. 2015;41:387-389.

36. Powell M, Miller R. Privatizing the English National Health Service: an irregular verb? J Health Polit Policy Law. 2013;38(5):1051-9.

37. Mays N. Introduction to the English health system and NHS: current reforms and 'hot' issues. London: UNC Gillings School of Global Public Health; 2014.

\author{
Endereço para correspondência: \\ Mariana Ribeiro Jansen Ferreira \\ Rua Monte Alegre, 984 \\ Bairro: Perdizes \\ CEP: 05014-901 - São Paulo - SP - Brasil \\ E-mail: mariana.jansen@gmail.com
}

